\section{MS8-P2 Structural Analysis of a Soluble Fragment of the Membrane Fusion Protein HlyD in a Type I Secretion System of Escherichia coli.}

Nam-Chul $\mathrm{Ha}^{1}$, Jin-Sook Ahn ${ }^{1}$

1. Department of Agricultural Biotechnology, CALS, Seoul National University

email: hanc210@snu.ac.kr

The protein toxin HlyA of Escherichia coli is exported without a periplasmic intermediate by the type I secretion system (T1SS). The T1SS is composed of an inner membrane ABC transporter HlyB, an outer-membrane channel protein $\mathrm{TolC}$, and a membrane fusion protein HlyD. However, the assembly of the T1SS remains to be elucidated. In this study, we determine the crystal structure of a part of the C-terminal periplasmic domain of HlyD. The long $\alpha$-helical domain consisting of three $\alpha$ helices and a lipoyl domain was identified in the crystal structure. Based on the HlyD structure, we modeled the hexameric assembly of HlyD with a long $\alpha$-helical barrel, which formed a complex with TolC in an intermeshing cogwheel-to-cogwheel manner, as observed in tripartite RND-type drug efflux pumps. These observations provide a structural blueprint for understanding the type I secretion system in pathogenic Gram-negative bacteria.

Keywords: HlyD, T1SS, Hexamer, Toxin secretion, E. coli

\section{MS8-P3 Structure of complete rotary ATP}

synthase and its role as new drug target against tuberculosis

\author{
Thomas Meier ${ }^{1}$
}

1. Imperial College London

email: t.meier@imperial.ac.uk

F F-ATP synthases are paradigmatic molecular machines, which use the transmembrane electrochemical ion gradient to power ATP synthesis. The enzymes belong to the class of rotary ATPases, which all share a common architecture principle, consisting of a rotor and stator entity. While ions are shuttled through the $\mathrm{F}$ complex of the enzyme, torque is generated at the rotor/stator and transferred to the $\mathrm{F}_{1}$-catalytic subunits for ATP synthesis. In the opposite direction, ATP hydrolysis can be used to drive ion pumping. I am going to present the structure of complete ATP synthase taking advantage of the combined approach of X-ray crystallography and cryo-electron microscopy. I will also focus on biochemical and structural investigations of the ATP synthase with respect to the development of new antibiotics in the fight against infectious diseases such as tuberculosis.

Keywords: Bioenergetics, X-ray crystallography, electron microscopy 